\title{
Individual cognitive behavioral therapy for insomnia in breast cancer survivors: a randomized controlled crossover pilot study
}

This article was published in the following Dove Press journal:

Nature and Science of Sleep

15 December 2009

Number of times this article has been viewed

\author{
Lavinia Fiorentino' \\ John R McQuaid ${ }^{2}$ \\ Lianqi Liu $^{3}$ \\ Loki Natarajan ${ }^{4}$ \\ Feng $\mathrm{He}^{4}$ \\ Monique Cornejo 3 \\ Susan Lawton ${ }^{3}$ \\ Barbara A Parker 6 \\ Georgia R Sadler ${ }^{5}$ \\ Sonia Ancoli-Israel ${ }^{3}$ \\ 'Cousins Center for \\ Psychoneuroimmunology, Semel \\ Institute for Neuroscience and \\ Behavior, University of California, \\ Los Angeles, CA, USA; ${ }^{2}$ Department \\ of Psychiatry, University of California, \\ San Francisco, CA, USA; ${ }^{3}$ Department \\ of Psychiatry, ${ }^{4}$ Department of Family \\ and Preventive Medicine, ${ }^{5}$ Department \\ of Surgery, University of California, \\ San Diego School of Medicine, La Jolla, \\ CA, USA; ${ }^{\circ}$ Moores UCSD Cancer \\ Center, La Jolla, CA, USA
}

Correspondence: Sonia Ancoli-Israel Professor of Psychiatry, Department of Psychiatry, University of California, San Diego, 9500 Gilman Drive \#0733, La Jolla, CA 92093-0733, USA

Tel +l 8588227710

Fax +I 8588227712

Email sancoliisrael@ucsd.edu
Purpose: Estimates of insomnia in breast cancer patients are high, with reports of poor sleep lasting years after completion of cancer treatment. This randomized controlled crossover pilot study looked at the effects of individual cognitive behavioral therapy for insomnia (IND-CBT-I) on sleep in breast cancer survivors.

Patients and methods: Twenty-one participants were randomly assigned to either a treatment group (six weekly IND-CBT-I sessions followed by six weeks of follow up) or a delayed treatment control group (no treatment for six weeks followed by six weekly IND-CBT-I sessions). Of these, 14 participants completed the pilot study (six in the treatment group and eight in the delayed treatment control group).

Results: Self-rated insomnia was significantly improved in the treatment group compared to the waiting period in the delayed treatment control group. The pooled pre-post-IND-CBT-I analyses revealed improvements in self-rated insomnia, sleep quality, and objective measures of sleep. Conclusions: These preliminary results suggest that IND-CBT-I is appropriate for improving sleep in breast cancer survivors. Individual therapy in a clinic or private practice may be a more practical option for this population as it is more easily accessed and readily available in an outpatient setting.

Keywords: insomnia, breast cancer, cognitive behavioral therapy

\section{Introduction}

Insomnia is common in breast cancer survivors, ${ }^{1}$ with estimates ranging from $19 \%{ }^{2}$ to $73 \%{ }^{3}$ Women with breast cancer are prone to insomnia for various reasons, including a general increase in psychological distress after the cancer diagnosis and disruption of sleep due to increased frequency and severity of hot flashes caused by menopause, which are often induced by the chemotherapy used for breast cancer treatment. ${ }^{4}$

Cognitive behavioral therapy for insomnia (CBT-I) is considered the gold standard treatment of primary and comorbid insomnia. ${ }^{5} \mathrm{CBT}-\mathrm{I}$ combines educational information with behavioral and cognitive strategies. Several studies examined cognitive behavioral interventions for insomnia in breast cancer survivors and reported improvements in sleep, quality of life (QoL), and psychological and immunological variables. ${ }^{6-11}$ Three $^{-}$ of these studies used traditional cognitive behavioral therapy (ie, inclusive of cognitive restructuring techniques as well as behavioral strategies), ${ }^{8-10}$ while the last study implemented a cognitive behavioral intervention consisting of education, stimulus control, sleep restriction, and sleep hygiene without formal cognitive restructuring techniques. $^{7}$ All the above studies implemented a group therapy modality, which is cost-effective and highly amenable to hospital settings. However, as most breast cancer submit your manuscript | www.dovepress.com

Dovepress 
survivors are community-dwelling, individual therapy in a clinic or private practice may be a more practical option for this population as it is more easily accessed and readily available in an outpatient setting.

This pilot study examined the effects of six individual sessions of cognitive behavioral treatment for insomnia (IND-CBT-I) on sleep. We hypothesized that: 1) subjective and objective measures of sleep would improve after IND-CBT-I compared to the no treatment; 2) the effects of IND-CBT-I on sleep would be maintained at six weeks follow-up.

\section{Methods}

\section{Participants}

Forty-four women who were breast cancer survivors were referred to the study from various sources including word of mouth, oncologists working in the San Diego community and the Rebecca and John Moores University of California; San Diego (UCSD) Cancer Center. Brochures and fliers promoting the study were distributed in wig and coffee shops in the San Diego community. An advertisement promoting the study appeared in the local newspaper and was posted on the internet (Craig's List and the Cancer Navigator websites). Twenty-one participants consented and were enrolled in the study. Because screening for eligibility was done after the randomization, five participants who had been randomized did not meet criteria for the study and hence were dropped. Fourteen participants finished the study (see Figure 1 for participants' flowchart).

All participants self-reported a history of breast cancer and had completed breast cancer treatment. The mean number of years since completion of the cancer treatment was six years (range of five months to 24 years, median five years; see Table 1 for participant cancer information).

\section{Protocol}

Study inclusion criteria consisted of meeting the Diagnostic Statistical Manual of Mental Disorders (DSM IV) ${ }^{12}$ criteria for insomnia and being a breast cancer survivor of any stage of cancer, who had completed breast cancer treatment (eg, surgery, radiation therapy, chemotherapy). Women on hormonal therapy were allowed in the study. Exclusion criteria included having current severe medical or psychiatric conditions that would interfere with the study protocol. Hypnotic use and sleep aids were not grounds for disqualification, although participants were asked not to increase dosage or change medications during the study. The use of medications and sleep aids was monitored throughout the study. This study was approved by the UCSD and the San Diego State University Committees on Human Research.
Screening assessments for eligibility were done with the semistructured clinical inventory for DSM-IV for axis I psychiatric screening, ${ }^{13}$ the sleep disorders symptom checklist, ${ }^{14}$ a medical inventory, and an unstructured clinical interview. The study had a cross-over design and was 13 weeks long. Participants were randomly assigned through a computer generated random list to one of two conditions: treatment condition (TxC) or delayed treatment control condition (DTxC). In the TxC condition, participants received six, weekly individual cognitive behavioral therapy for insomnia (IND-CBT-I) sessions followed by six weeks of no-treatment follow-up. In the DTxC, participants were first followed for six weeks of continued usual care (ie, they were advised to continue doing whatever they were doing to manage their insomnia without changing dosage or frequency of treatment) followed by the six weekly individual sessions of IND-CBT-I. Data were collected at baseline (Phase 0 ), at the end of the first six-weeks (Phase 1; end of treatment for the TxC and the end of six weeks of usual care for the DTxC), and at the end of the second lot of six weeks (Phase 2; end of six-week follow-up for the TxC and end of six-weeks of IND-CBT-I treatment for the DTxC).

Demographic and cancer diagnosis information were collected at phase 0 (Table 1). The assessments for medication intake and insomnia were collected at each phase $(0,1$, and 2) as were the 72 hours of objective measures of sleep via actigraphs. In addition, weekly sleep quality assessments were collected for all 13 weeks (baseline +12 weeks) of the study, and daily sleep diary data were collected during the treatment phase.

\section{Treatment}

The manualized IND-CBT-I treatment consisted of six one-hour, weekly sessions of individual IND-CBT-I. ${ }^{14}$ The IND-CBT-I was comprised of education (both the nature of insomnia and cognitive behavioral therapy basics), behavioral components (sleep restriction, stimulus control, adhering to the sleep hygiene rules and training in progressive muscle relaxation techniques ${ }^{15}$ ) and cognitive interventions (challenging the dysfunctional beliefs about sleep and any other belief or thought that kept the patient awake at night together with restructuring the maladaptive thoughts into more realistic, functional, and less anxiety provoking thoughts). Examples of maladaptive thoughts encountered were: "If I don't get a full night of sleep (eight hours) my cancer will come back," and "I should always be happy and grateful because I've been given a second chance in life." The IND-CBT-I also entailed homework assignments including sleep diaries and practicing the behavioral and 


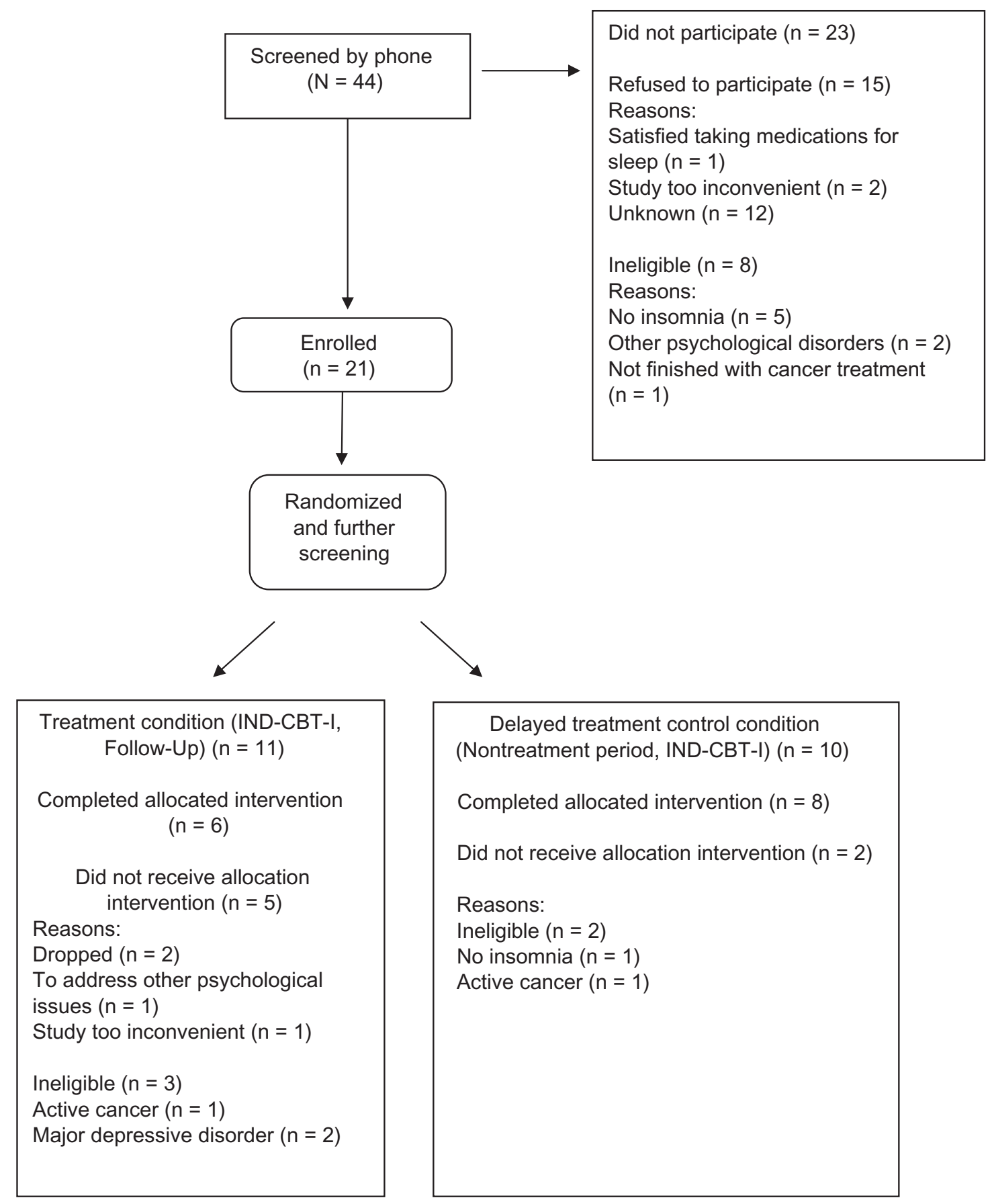

Figure I Participant flowchart.

Abbreviation: IND-CBT-I, individual cognitive behavioral therapy for insomnia.

cognitive strategies (eg, scheduling worry time by setting a time-limited period to worry every day). All participants received all components of the treatment.

\section{Measures}

\section{Actigraphy}

Objective measures of sleep were collected with actigraphy.

The Actillume (Ambulatory Monitoring Inc, Ardsley, NY), a small device, measuring approximately $1 \times 3 \times 6 \mathrm{~cm}$, was worn on the nondominant wrist. The Actillume ${ }^{\circledR}$ contains a piezoelectric linear accelerometer (sensitive to $0.003 \mathrm{~g}$ and above), a microprocessor, 32K RAM memory, and associated circuitry. Data were sampled every second and stored every minute. In addition to wearing the Actillume, the participants filled out a daily paper and pencil log with information about their time in bed, time out of bed, naps and times when they 
Table I Participant cancer diagnosis and treatment summary

\begin{tabular}{ll}
\hline Original diagnosis & Number \\
\hline Invasive lobular carcinoma & 4 \\
Invasive ductal carcinoma & 3 \\
Mixed ductal and lobular carcinoma & 2 \\
Other pathology & 3 \\
Unknown & 2 \\
Cancer stage & \\
Stage I & 7 \\
Stage II & 3 \\
Stage III & 2 \\
Stage III-advanced & 2 \\
Surgery & \\
Lumpectomy & 8 \\
Mastectomy & 3 \\
Double mastectomy & 2 \\
Other therapy & \\
Chemotherapy & 7 \\
Radiation therapy & 11 \\
Hormonal therapy & 8 \\
\hline
\end{tabular}

Notes: Some women received combinations of therapy: three participants received chemotherapy, radiation therapy, and hormonal therapy; three participants received chemotherapy and radiation therapy; three participants received radiation therapy and hormonal therapy; and one participant received chemotherapy and hormonal therapy.

took the Actillume off (eg, for showering), and log data were used to edit the actigraphic records. Variables analyzed were: total sleep time (TST); wake after sleep onset (WASO); number of awakenings per night; and percent sleep (percent of time asleep at night/time in bed).

\section{Insomnia}

Insomnia severity was assessed with the insomnia severity index (ISI). ${ }^{16}$ The ISI is an insomnia severity questionnaire that assesses seven aspects of insomnia: difficulty falling asleep; nighttime awakenings; early morning awakenings; impairment of daytime functioning; noticeability of impairments; distress and worry about sleep and current dissatisfaction with sleep. Each item is rated on a Likert scale ranging from zero (not at all) to four (extremely) and scores are added to compute the ISI total score (range 0-28). The ISI internal consistency is high (ie, 0.78). Scores of eight and above are considered clinically significant insomnia.

\section{Sleep quality}

The Pittsburgh sleep quality index (PSQI) measures reported sleep patterns and sleep problems, including sleep quality, sleep latency, sleep efficiency, and napping behavior. ${ }^{17}$ The PSQI is a 19-item questionnaire that has been demonstrated to have high internal consistency ( 0.83$)$, test-retest reliability (0.85) and diagnostic validity. Scores from subscales are added to compute a global score of overall quality of sleep over the prior one-week period (Daniel Buysse, pers comm). Global sleep quality scores are continuous (range $0-21$ ) with high scores reflecting poor sleep quality. A score above eight is considered poor sleep in clinical cancer populations. ${ }^{18}$

\section{Sleep diaries}

A daily sleep diary was completed by each participant during the first five weeks of the IND-CBT-I. The variables extracted from the sleep diaries were time in bed (TIB), total sleep time (TST), WASO, sleep onset latency (SOL, the time it takes to fall asleep), sleep efficiency $(\mathrm{SE}=(\mathrm{TST} / \mathrm{TIB}) \times 100)$, and number of wakes.

\section{Sleep medications}

The number of medications used to aid sleep was assessed by having participants, at each phase of the study, complete a form which listed various types of medications and asked whether that medication was taken to aid their sleep during the past week.

\section{Data analyses}

The actigraphy data were downloaded onto a desktop computer and edited based on sleep logs by a trained research staff member blind to the study condition of the participants. The Action-4 software package (Ambulatory Monitoring Inc) was used to score sleep and wake. Statistical analyses were performed using SPSS software (version 15; SPSS Inc, Chicago, IL). ${ }^{19}$ Because of the small sample size and violation of the assumptions needed for parametric statistics, differences between groups on demographic and cancer diagnosis and treatment variables were tested using the Mann-Whitney $U$ nonparametric independent sample tests. Mann-Whitney $U$ tests were also used to look at the differences in change scores (calculated by subtracting the phase 1 scores from the phase 0 baseline scores) between the $\mathrm{TxC}$ group and the DTxC group.

To examine differences before and after treatment, data from all participants $(\mathrm{N}=14)$ were analyzed using the nonparametric Wilcoxon signed rank tests for paired analyses. The Wilcoxon signed rank test was also used to examine differences in sleep diary variables from session 1 to session 5 (baseline and post-session 6 sleep diary data were not collected). Finally, the Wilcoxon signed rank test was used to examine differences between pre-IND-CBT-I, post-IND-CBT-I and follow-up in the $\mathrm{TxC}$ group. 
To examine the effect size of the treatment, Cohen's d estimates were computed by using the mean change scores of the $\mathrm{TxC}$ and DTxC groups from phase 0 to phase 1 and the pooled standard deviation for the questionnaires and actigraphy variables, and using the pre-post-treatment means and pretreatment standard deviation for the sleep diary variables..$^{20}$

\section{Results}

\section{Demographic information}

Fourteen women (Mean age 61 years, standard deviation $(\mathrm{SD})=11.6$; range $=45-85$ years) completed the study. Twelve participants were Caucasian, one was Asian, one reported more than one race, and one participant was Hispanic.

There were no statistically significant differences at phase 0 between the TxC and the DTxC groups on demographic variables (age, income, education), sleep medications, cancer treatment variables (type of treatment, stage), or objective sleep variables.

\section{Objective measures of sleep}

There were no significant differences in the change scores analyses between the TxC and DTxC groups at phase 1 . However, the pooled analyses revealed that after the six sessions of IND-CBT-I there were statistically significant decreases in TST, WASO, number of wakes and statistically significant increases in sleep percentages (Table 2). In addition the analysis of the TxC group six-week follow-up data showed that improvements were maintained for WASO (Phase 0: $M=1.41, S D=0.73$, range $=0.69-2.23$; Phase 2: $M=1.03$, $S D=0.52$, range $=0.4-1.7, P=0.043)$, and for number of wakes (Phase $0: M=23, S D=8.88$, range $=14-36$; Phase 2 : $M=20, S D=8.44$, range $=9-31, P=0.043$ ).

\section{Subjective measures of sleep Insomnia severity index}

As shown in Figure 2, at Phase 0, although both groups scored in the range suggesting clinically significant insomnia, participants in the TxC group had statistically significant lower scores on the ISI than those in the DTxC group $(P=0.04)$.

The analysis of the change scores from phase 0 to phase 1 revealed a significant decrease in insomnia in the TxC group compared to the DTxC group $(P=0.03)$ (Figure 2). In addition, the pooled analyses revealed significantly less insomnia post-IND-CBT-I compared to pre-IND-CBT-I $(P=0.001)$. At the six week follow-up, the participants in the TxC group continued to improve, reporting less insomnia compared to phase $1(P=0.03)$. Clinically significant reductions of insomnia severity (scores $<9=$ no insomnia) were found for six out of the 14 participants after IND-CBT-I. The Cohen's d effect size estimate for the ISI was large $(\mathrm{d}=1.4){ }^{20}$

\section{Pittsburgh sleep quality index}

Parallel to the ISI, both groups scored in the range suggesting clinically relevant sleep disruption at phase 0 , with participants in the TxC group reporting significantly better sleep quality compared to participants in DTxC group $(P=0.008)$ (Figure 3). The analysis of the change scores from phase 0 to phase 1 revealed no significant differences. However, the pooled analyses revealed significantly better sleep quality Post-IND-CBT-I compared to Pre-IND-CBT-I $(P=0.002)$. The results were maintained at the six week follow-up for the $\operatorname{TxC}(P=0.046)$. Clinically significant reductions of sleep disturbance (scores $\leq 8$ ) were found for 10 of the 14 participants after IND-CBT-I. The Cohen's d effect size estimate for the PSQI was large $(\mathrm{d}=0.8) \cdot{ }^{20}$

\section{Sleep diaries}

The pooled data from all 14 participants showed statistically significant improvements at week 5 of IND-CBT-I compared to week 1 in WASO, SE and number of wakes (Table 3). The Cohen's d effect size estimates ranged from small (eg, TST) to large (eg, SE) (Table 3). ${ }^{20}$

Table 2 Actigraphic variables before and after IND-CBT-I

\begin{tabular}{|c|c|c|c|c|c|c|c|c|}
\hline & \multicolumn{3}{|c|}{ Pre-Tx $n=14$} & \multicolumn{3}{|c|}{ Post-six weeks of Tx $n=14$} & \multirow[t]{2}{*}{ P-value } & \multirow[t]{2}{*}{ Cohen's d } \\
\hline & Mean & $S D$ & Range & Mean & $S D$ & Range & & \\
\hline TST (h) & 7.26 & 0.76 & $6-8.5$ & 6.64 & $\mathrm{I} .4 \mathrm{I}$ & $4.7-9.7$ & 0.03 & -0.5 \\
\hline WASO (h) & 1.63 & 0.66 & $0.7-2.5$ & 1.04 & 0.31 & $0.6-1.6$ & 0.009 & 0.6 \\
\hline \#Wakes & 26 & 7.8 & $14-36$ & 23 & 6.3 & $13-33$ & 0.046 & 0.2 \\
\hline Sleep \% & 82 & 6 & $74-92$ & 86 & 3 & $79-92$ & 0.02 & 5.6 \\
\hline
\end{tabular}

Notes: Data analyses using Wilcoxon signed rank test. Cohen's d calculated on differences between mean change scores of the TxC and DTxC groups from phase 0 to phase I. Abbreviations: IND-CBT-I, individual cognitive behavioral therapy for insomnia; TxC, treatment condition; ISI, insomnia severity index; TxC, treatment + follow-up group; DTxC, waiting + treatment group; N, number; M, mean; SD, standard deviation; TST, total sleep time; WASO, wake time after sleep onset; H, hours. 


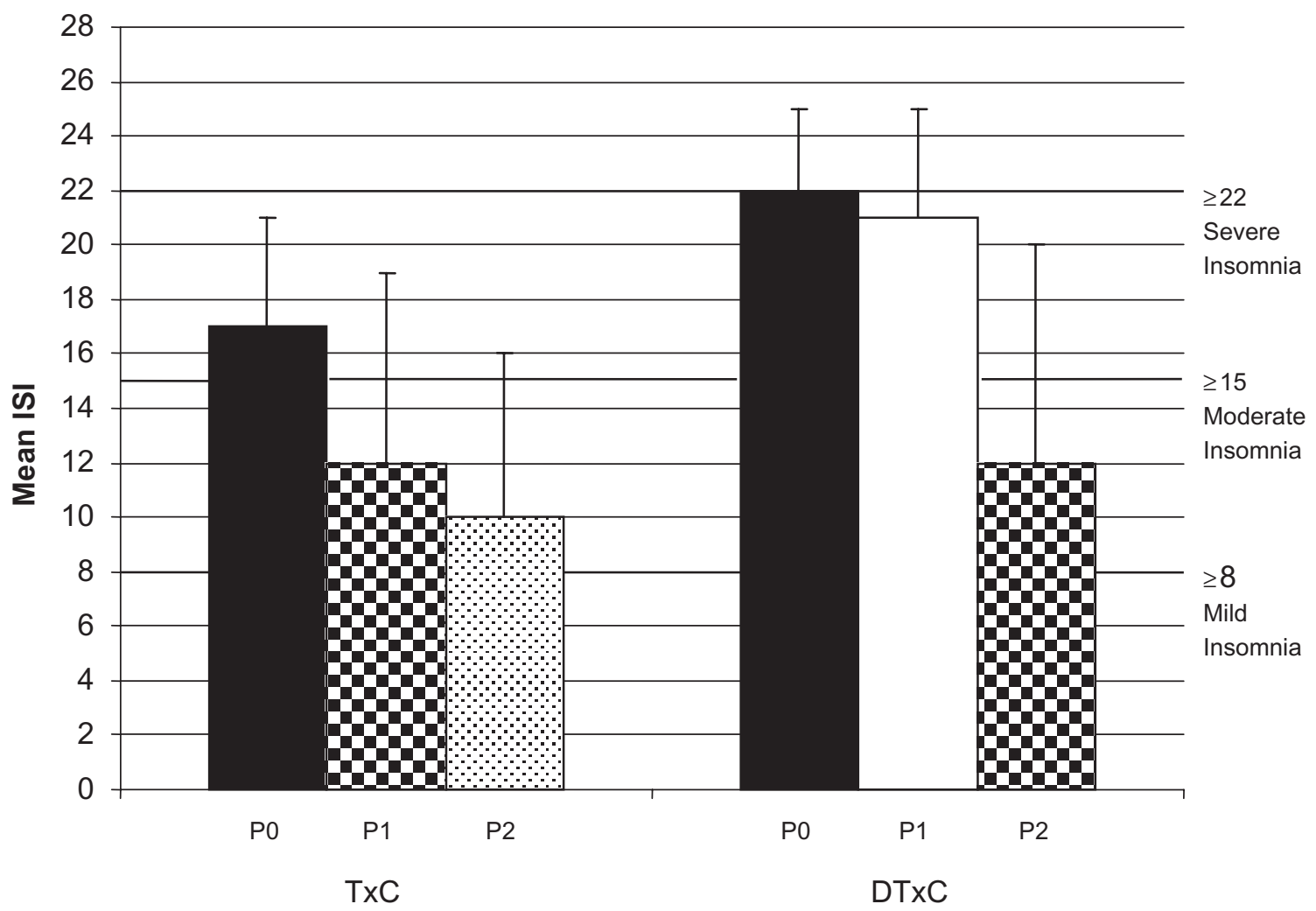

$\begin{array}{llll}\text { Baseline } & \text { Post CBT-I } & \text { Follow up } \quad \square & \text { Post-waiting }\end{array}$

Figure 2 Insomnia severity index by group (means and standard deviations).

Abbreviations: IND-CBT-I, individual cognitive behavioral therapy for insomnia; TxC, treatment condition; ISI, insomnia severity index; TxC, treatment + follow-up group; DTxC, waiting + treatment group; P, phase.

\section{Sleep medications}

There were no differences in medication use at phase 0 between the groups, and there were no differences in the change scores analyses between the TxC and DTxC groups in amount of sleep medication used. The pooled analyses revealed a statistically significant decrease in sleep medication use post-IND-CBT-I compared to pre-IND-CBT-I (Pre-IND-CBT-I: $M=1.00$, Median $=1, S D=1.46$, range $=$ $0-5$; post-IND-CBT-I: $M=0.07$, Median $=0, S D=0.27$, range $=0-1, z=-2.264, P=0.02)$. The Cohen's d effect size estimate was medium $(\mathrm{d}=0.4) .^{20}$

\section{Discussion}

To our knowledge this is the first empirical study of individual CBT-I in breast cancer survivors that examined changes in sleep. The results of this study suggest that both subjective and objective measures of sleep disturbance, as well as ratings of insomnia severity, improved after six weeks of individual IND-CBT-I. Furthermore, consistent with the participants' sleeping better, the use of medications to aid their sleep decreased after treatment. The ISI and PSQI post treatment assessments revealed that the improvements were clinically significant: Insomnia was completely resolved in $43 \%$ of the participants and sleep disturbance was completely resolved in $71 \%$ of the participants.

There was a statistically significant decrease in actigraphic-measured TST after IND-CBT-I, which may seem paradoxical for an insomnia treatment study, but is consistent with the prescription of sleep restriction and stimulus control therapy which decrease the opportunity for sleep, with the intent of improving sleep consolidation. In fact, although TST decreased, WASO and number of wakes also decreased after IND-CBT-I, and sleep percent increased, supporting the notion that the participants' sleep was more consolidated.

One of the benefits of the individual therapy setting was the amount of individualized treatment and attention the participants received. This was particularly salient in the cognitive sessions, where participants tackled specific maladaptive thoughts affecting their sleep. These thoughts 


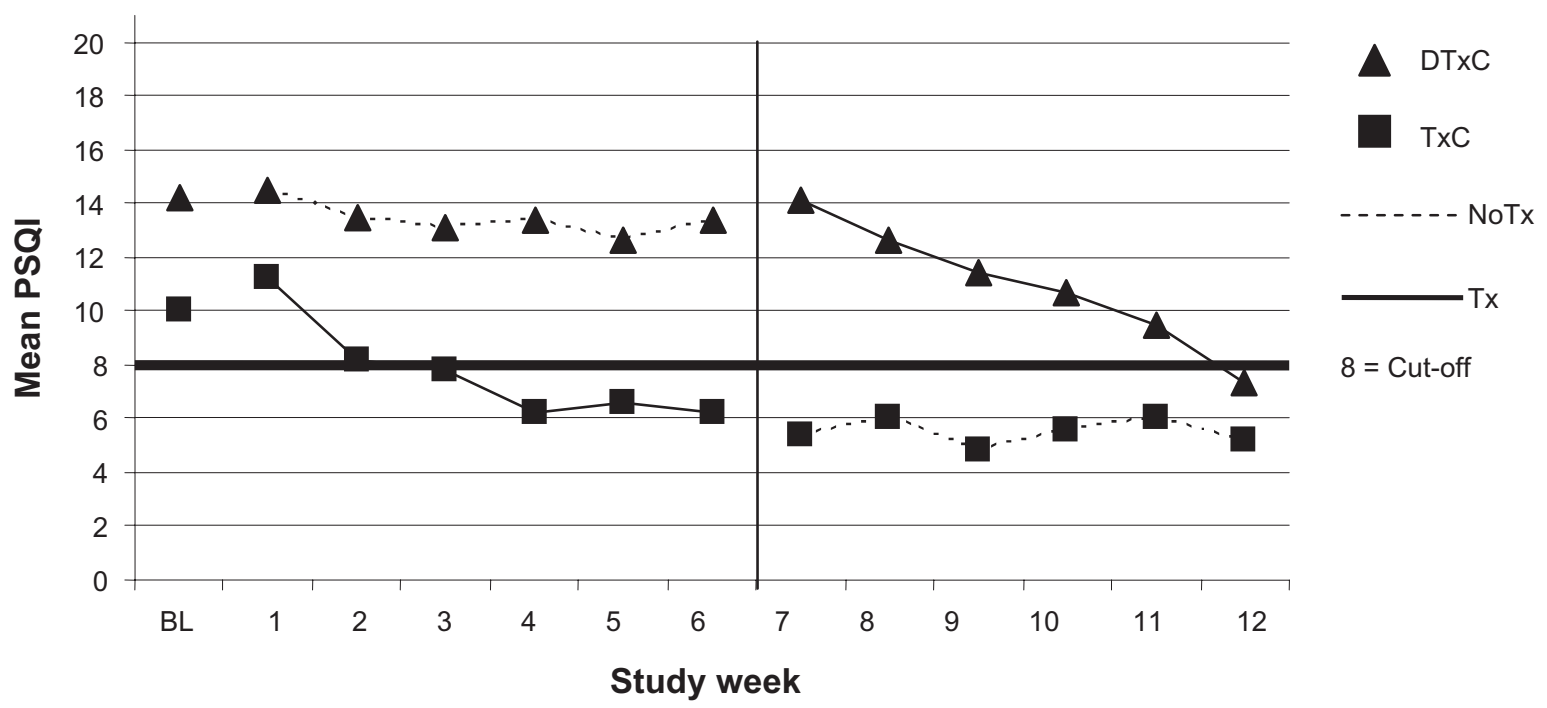

Figure 3 Pittsburgh sleep quality index by group and week of study.

Abbreviations: PSQI, Pittsburgh sleep quality index; BL, baseline [ie, phase 0]; IND-CBT-I, individual cognitive behavioral therapy for insomnia; TxC, treatment condition; ISI, insomnia severity index; TxC, treatment + follow-up group; DTxC, waiting + treatment group.

provided valuable information to efficiently direct the trajectory of the treatment. One woman's comments revealed surprisingly negative attitudes towards sleeping which began after being diagnosed with breast cancer ("When I was diagnosed with breast cancer, I thought I was going to die. I never wanted to go to bed and sleep, because for me sleep was like not-living and I wanted to live as much as possible. ... Then, when I was cured, I realized that I should sleep, but I had forgotten how to do it.") The degree to which beliefs and attitudes contribute to sleep problems in cancer patients in unknown, but is worth exploring in future research as it may inform the development of insomnia prevention programs in breast cancer and other life-threatening medical conditions.

A limitation of this pilot study was the small sample size which likely underpowered our statistical analysis of the differences between the $\mathrm{TxC}$ and the $\mathrm{DTxC}$ groups at phase 1 for the sleep disturbance variables. The small sample size also limited the racial and ethnic groups represented in the sample, and decreased the generality of the findings to diverse populations. It is important to note that the small sample size was not a consequence of treatment burden, but of the nature of the study (a pilot), and of the participants not meeting the eligibility requirements (after the post randomization screening). Because of the small sample size and low power statistical analysis, we were not able to statistically correct for testing multiple outcome measures and hence have an increased type I error rate in this study. However, we hypothesized that specific sleep measures (ISI, PSQI, and objective sleep) would improve and, despite the lowered power to detect effects, we were able to test and confirm this, which speaks to the strength of the IND-CBT-I treatment which was also confirmed by the large effect size estimates yielded on the insomnia and sleep disruption variables.

Table 3 Sleep diary data before and after IND CBT-I

\begin{tabular}{|c|c|c|c|c|c|c|c|c|}
\hline & \multicolumn{3}{|c|}{ Session I $N=14$} & \multicolumn{3}{|c|}{ Session $5 N=14$} & \multirow[t]{2}{*}{$\mathbf{P}$} & \multirow[t]{2}{*}{ Cohen's c } \\
\hline & Mean & $S D$ & Range & Mean & $S D$ & Range & & \\
\hline WASO (m) & 53.4 & 42.98 & $3-164$ & 25.20 & 25.64 & $0-96$ & 0.002 & 0.6 \\
\hline SE (\%) & 73.09 & 15.00 & $35-92$ & 83.25 & 10.96 & $57-98$ & 0.01 & 0.7 \\
\hline \# Wakes & 3 & 1.3 & $1-6$ & 2 & 2 & $0-7$ & 0.02 & 0.6 \\
\hline TST (m) & 345 & 78 & I78-504 & 347 & 66 & $242-454$ & 0.78 & 0.02 \\
\hline SOL (m) & 44 & 41 & $5-143$ & 25 & 23 & $3-81$ & 0.11 & 0.5 \\
\hline
\end{tabular}

Notes: Data analyses using Wilcoxon signed rank test. Cohen's $\mathrm{d}$ calculation based on differences of means before and after treatment.

Abbreviations: IND-CBT-I, individual cognitive behavioral therapy for insomnia; TxC, treatment condition; ISI, insomnia severity index; TxC, treatment + follow-up group; DTxC, waiting + treatment group; N, number; SD, standard deviation; TST, total sleep time; WASO, wake time after sleep onset; h, hours; SOL, sleep onset latency; M, minutes. 


\section{Conclusion}

This is the first study in breast cancer that has used individual CBT-I instead of group therapy, and hence informs the literature on the feasibility and possible advantages of this therapeutic form for this population. These preliminary findings show that individual IND-CBT-I is feasible, easily transportable to nonhospital settings such as private practitioners offices, and is beneficial in decreasing levels of insomnia in breast cancer survivors. Increased awareness of behavioral treatments for insomnia, as well as the availability of professionals trained in the administration of individual CBT-I in private practice and community clinics are important factors that will affect breast cancer survivors' access to this valuable insomnia treatment option.

\section{Acknowledgments}

The authors would like to thank Matthew Marler, PhD, for consultation during the initial design phase of the study. This research was supported by CBCRP 11GB-0049, NCI CA112035, CBCRP 11IB-0034, P60MD00220, NCI P30 CA023100-22, T32-MH19925 Moores UCSD Cancer Center, the Research Service of the VASDHS, and the Cousins Center for Psychoneuroimmunology, UCLA. Dr Ancoli-Israel has received grant support from Sepracor Inc and Litebook Inc., and equipment from Respironics. She has consulted for Ferring Pharmaceuticals Inc., GlaxoSmithKline, Pfizer, Respironics, sanofi-aventis, Schering-Plough. No other authors have any disclosures or report any conflicts of interest.

\section{References}

1. Fiorentino L, Ancoli-Israel S. Insomnia and its treatment in women with breast cancer. Sleep Med Rev. 2006;10(6):419-429.

2. Savard J, Simard S, Blanchet J, Ivers H, Morin CM. Prevalence, clinical characteristics, and risk factors for insomnia in the context of breast cancer. Sleep. 2001;24(5):583-590.

3. Carpenter JS, Elam JL, Ridner SH, Carney PH, Cherry GJ, Cucullu HL. Sleep, fatigue, and depressive symptoms in breast cancer survivors and matched healthy women experiencing hot flashes. Oncol Nurs Forum. 2004;31(3):591-598.
4. Kryger MH. A women's guide to sleep disorders. New York, NY: McGraw-Hill, 2004.

5. National Institutes of Health State of the Science Conference Statement on Manifestations and Management of Chronic Insomnia in Adults. Sleep. 2005;28(9):1049-1058.

6. Dirksen SR, Epstein DR. Efficacy of an insomnia intervention on fatigue, mood andquality of life in breast cancer survivors. J Adv Nurs. 2008;61(6):664-675.

7. Epstein DR, Dirksen SR. Randomized trial of a cognitive-behavioral intervention for insomnia in breast cancer survivors. Oncol Nurs Forum. 2007;34(5):E51-E59.

8. Espie CA, Fleming L, Cassidy J, et al. Randomized controlled clinical effectiveness trial of cognitive behavior therapy compared with treatment as usual for persistent insomnia in patients with cancer. $J$ Clin Oncol. 2008;28(28):1-9.

9. Quesnel C, Savard J, Simard S, Ivers H, Morin, CM. Efficacy of cognitive-behavioral therapy for insomnia in women treated for nonmetastatic breast cancer. J Consult Clin Psychol. 2003;71(1): 189-200.

10. Savard J, Simard S, Ivers H, Morin CM. Randomized study on the efficacy of cognitive-behavioral therapy for insomnia secondary to breast cancer, part I: Sleep and psychological effects. J Clin Oncol. 2005;23(25):6083-6096.

11. Savard J, Simard S, Ivers H, Morin CM. Randomized study on the efficacy of cognitive-behavioral therapy for insomnia secondary to breast cancer, part II: Immunologic effects. J Clin Oncol. 2005;23(25):6097-6106.

12. American Psychiatric Association. Diagnostic and Statistical Manual of Mental Disorders, Fourth Edition, Text Revision: DSM-IV-TR. Washington, DC: American Psychiatric Association, 2000.

13. Michael B First, Robert L Spitzer, Miriam Gibbon, Janet BW. Williams Structured Clinical Interview for DSM-IV Axis I Disorders (SCID-I), Clinician Version, User's Guide. Washington, DC: American Psychiatric Association; 1997.

14. Perlis ML, Jungquist C, Smith MT, Posner D. Cognitive Behavioral Treatment of Insomnia. New York, NY: Springer; 2005.

15. Bernstein DA, Borkovec TD, Hazlett-Stevens H. New Directions in Progressive Relaxation Training: A guidebook for helping professionals. Westport, CT: Praeger Publishers; 2000.

16. Bastien CH, Vallieres A, Morin CM. Validation of the Insomnia Severity Index as an outcome measure for insomnia research. Sleep Med. 2001; 2(4):297-307.

17. Buysse DJ, Reynolds CF III, Monk TH, Berman SR, Kupfer DJ. The Pittsburgh Sleep Quality Index: a new instrument for psychiatric practice and research. Psychiatry Res. 1989;28(2):193-213.

18. Carpenter JS, Andrykowski MA. Psychometric evaluation of the Pittsburgh Sleep Quality Index. J Psychosom Res. 1998;45:5-13.

19. Statistical Package for the Social Sciences. SPSS for Windows, Version 15.0.0. Chicago, IL: SPSS, Inc; 2006

20. Cohen J. Statistical Power Analysis for the Behavioral Sciences (2nd ed.). Hillsdale, NJ: Lawrence Earlbaum Associates; 1988.
Nature and Science of Sleep

\section{Publish your work in this journal}

Nature and Science of Sleep is an international, peer-reviewed, open access journal covering all aspects of sleep science and sleep medicine, including the neurophysiology and functions of sleep, the genetics of sleep, sleep and society, biological rhythms, dreaming, sleep disorders and therapy, and strategies to optimize healthy sleep. The journal welcomes

\section{Dovepress}

original research, clinical \& epidemiological studies, reviews \& evaluations, case reports and extended reports. The manuscript management system is completely online and includes a very quick and fair peerreview system, which is all easy to use. Visit http://www.dovepress.com/ testimonials.php to read real quotes from published authors. 